\title{
BMJ Open Virtual communities of practice to improve clinical outcomes in healthcare: protocol for a 10-year scoping review
}

\author{
Louise Shaw (D) , ${ }^{1,2}$ Dana Jazayeri, ${ }^{2}$ Debra Kiegaldie, ${ }^{1,3}$ Meg Morris (iD) ${ }^{2,4}$
}

To cite: Shaw L, Jazayeri D, Kiegaldie D, et al. Virtual communities of practice to improve clinical outcomes in healthcare: protocol for a 10year scoping review. BMJ Open 2021;11:e046998. doi:10.1136/ bmjopen-2020-046998

- Prepublication history and additional supplemental material for this paper are available online. To view these files, please visit the journal online (http://dx.doi.org/10.1136/ bmjopen-2020-046998)

Received 15 November 2020 Accepted 04 May 2021

Check for updates

(C) Author(s) (or their employer(s)) 2021. Re-use permitted under CC BY-NC. No commercial re-use. See rights and permissions. Published by BMJ.

${ }^{1}$ Faculty of Health Science, Youth \& Community Studies, Holmesglen Institute of TAFE, Parkdale, Victoria, Australia ${ }^{2}$ School of Allied Health, Human Services and Sport, Faculty of Science, Health and Engineering, La Trobe University, Bundoora, Victoria, Australia

${ }^{3}$ Eastern Clinical School, Faculty of Medicine, Nursing \& Health Sciences, Monash University, Melbourne, Victoria, Australia

${ }^{4}$ Victorian Rehabilitation Centre, Healthscope Limited, Melbourne, Victoria, Australia

Correspondence to Dr Louise Shaw; louise.shaw@holmesglen. edu.au

\section{ABSTRACT}

Introduction Virtual communities of practice (VCoPs) use a common online platform to provide healthcare professionals with the opportunity to access highly specialised knowledge, build a professional support network and promote the translation of research evidence into practice. There is limited reporting of how best to design and administer VCoPs within healthcare organisations. The primary aim of this scoping review is to identify the best methods used to establish and maintain VCoPs. Findings shall be used to develop a flexible framework to guide the establishment and facilitation of a $\mathrm{VCOP}$ for healthcare professionals to ensure the translation of falls prevention clinical guidelines into practice.

Methods and analysis A five-stage scoping review process will be followed based on Arksey and 0'Malley's framework and refined by the Joanna Briggs Institute Methodology. An initial limited search of PubMed and Cumulative Index to Nursing and Allied Health Literature will identify relevant studies and assist with search term development. This will be followed by a search of five online databases to identify papers published from January 2010 until November 2020. Papers will be independently screened by two reviewers, and data extracted and analysed using a reporting framework. Qualitative data will be analysed thematically and numerical synthesis of the data will be conducted.

Results and dissemination The results of this scoping review will highlight the best ways to design and manage VCoPs in healthcare organisations. The findings will be presented at relevant stakeholder workshops, conferences and published in peer-reviewed journals.

\section{INTRODUCTION}

Communities of practice (CoPs) within healthcare involve groups of people who share an interest in a particular topic, and a desire to deepen their knowledge and expertise by interacting with others regularly. ${ }^{12}$ They foster mutual learning and knowledge sharing outside the silos of discipline-specific professional expertise, ${ }^{3}$ provide a forum for developing and implementing evidencebased practice, ${ }^{4}$ and facilitate the delivery of high quality, cost-effective care. The three main elements characterising CoPs identified by Wenger et $a l^{1}$ are community (collective

\section{Strengths and limitations of this study}

The scoping review will identify methods used to establish and maintain virtual communities of practice (VCoPs) in healthcare.

- The review will provide detailed analysis of the extent of the literature on VCoPs in healthcare published in the last 10 years.

- The review will be limited to studies in English written in the last 10 years.

- VCoPs that are purely for teaching purposes, for example, online learning, will be excluded.

learning through social interactions), domain (within a particular area of interest) and practice (developing, sharing and maintaining knowledge). Examples of CoPs where professionals have sought further education, development and innovation in a particular practice area, include the promotion of a new measurement tool in child and youth mental healthcare, ${ }^{5}$ promotion of recoveryoriented practices in mental healthcare ${ }^{6}$ and the management of COVID-19. ${ }^{7}$

The advantages of CoPs within healthcare include the joint analysis of practical experiences and information among their members. ${ }^{3}$ They allow members to openly discuss concerns and acknowledge errors, encourage in-situ learning, shared decision-making and coordination of experimentation. ${ }^{8}$ While CoPs aim to promote standardisation of practice and the establishment of interpersonal relationships that encourage knowledge sharing, there is diversity in how and why they are implemented. ${ }^{9}$ CoPs in healthcare have been found to be complex and multifaceted. They vary in composition, intended purpose and use a variety of models for members to share their knowledge. ${ }^{9}$ The diversity of CoPs, can be influenced by various social, cultural and individual factors, such as clinical leadership, support and commitment for quality management, regular communication, and availability of accurate and relevant 
data. ${ }^{10}$ Their establishment requires a flexible framework that will guide their formation and ongoing operational procedures. ${ }^{9}$

Advances in technology-based communication and the growth of the internet has led to a rapid increase in the sharing of health information globally. Health professionals can use virtual communities of practice (VCoPs) to share their knowledge. ${ }^{11} 12$ More recently, the COVID-19 pandemic has significantly limited physical interactions and meetings for sharing of expertise, and therefore, the relevance and utility of VCoPs is more evident. ${ }^{13}$ VCoPs provide the opportunity to stay connected and informed, by the sharing of emerging resources and dissemination of research on health issues. ${ }^{13}$ VCoPs use a wide variety of media to establish a virtual collaborative space including social media sites, videoconferencing and websites. ${ }^{14}$ The creation of VCoPs means that health professionals who are geographically dispersed, ${ }^{15}$ can use virtual communities for learning, support, continuing professional education, knowledge management and information sharing. ${ }^{11} 12$ Being a member of a VCoP can be a great opportunity for healthcare professionals to share and gain access to highly specialised knowledge. ${ }^{16}$ They allow healthcare professionals to build a professional support network and promote the translation of evidence into daily practice, by accessing a common platform. ${ }^{17} 18$ VCoPs have a key role in promoting interprofessional learning and collaboration, with virtual modes of communication helping to reduce professional barriers and encourage communication within and between healthcare professions. ${ }^{19}$

The successful design and management of VCoPs depend on the characteristics of the virtual community. ${ }^{8}$ Members of CoPs and VCoPs are likely to experience very different environments because of the primary way they interact. ${ }^{14}$ Computer-mediated interactions are likely to make it more difficult for members to build mutual knowledge, trust, a sense of belonging and open exchange of ideas. ${ }^{2021}$ Factors found to affect knowledge sharing in online communities identified in the literature include individual factors, technological factors and social factors. ${ }^{22-25}$ Individual factors include the contributions of members, with active participation being essential for the VCoP to grow and develop. ${ }^{16}$ Active participation refers to members' knowledgeexchange activities, such as posting questions on online community boards, engaging in live chats, participating in online and videoconferencing discussion sessions and providing asynchronous answers and feedback in discussion threads. ${ }^{2627}$ Active participation is influenced by members' motivations, personalities, time available and values. ${ }^{1} 16$ 26-28 Social factors include the social interaction among members within the group and the roles of group moderators, while technological factors involve technical and usability issues. ${ }^{16}$ A 7-year longitudinal study by Antonacci et $a l^{16}$ showed the growth of VCoPs for healthcare professionals to be related to the presence of a centralised leadership structure and the frequent rotating of leadership over time.

Healthcare organisations have a responsibility to deliver high-quality, cost-effective care by implementing evidence-informed policy and practice. ${ }^{29-31}$ Despite the growing number of clinical guidelines produced by government agencies to improve effectiveness and quality of care ${ }^{32}$ frequently there are gaps between research evidence and clinical practice ${ }^{33-36}$ By providing a platform for healthcare professionals to collaborate towards a common purpose, VCoPs can bridge the gap between research evidence, policy-making and implementation of clinical guidelines. ${ }^{37}$ To attempt to address the problems of translating falls prevention clinical guidelines into practice across multiple sites of a residential aged care organisation, one team used a webbased falls prevention $\mathrm{CoP}^{28}$ Member engagement with the Information Communication Technology (ICT) applications of asynchronous discussions and accessing evidence were low, with a number of barriers and facilitators to web-based CoP operation identified. ${ }^{28}$ Barriers to sustainability included members' capabilities for using ICT applications and lack of dedicated time provided by management for web-based participation. ${ }^{28}$ However, the operation of a VCoP in falls prevention was found to be achievable if staff were given sufficient time, and provided with suitable training and support. ${ }^{38}$ All of these points could be considered when establishing a VCoP in falls prevention.

It is essential to clarify effective methods of VCoPs for knowledge synthesis and translation into practice. Given the limited reporting of a standard approach to the design and administration of VCoPs within healthcare, a scoping review shall be conducted to determine the nature of reported VCoPs within this context in the last 10 years. It aims to identify the methods used to establish and maintain VCoPs and ascertain potential barriers and facilitators to the implementation of VCoPs. This information will then be used to develop a flexible framework that will guide the establishment and facilitation of a VCoP for healthcare professionals on falls prevention in hospitals to assist the translation of clinical guidelines into practice.

\section{METHODS AND ANALYSIS}

The methodological structure will follow Arksey and O'Malley's framework for scoping reviews, ${ }^{39}$ which was refined by the Joanna Briggs Institute. ${ }^{40} 41$ The protocol will use the Preferred Reporting Items for Systematic reviews and Meta-Analyses extension for Scoping Reviews (PRISMA-ScR) checklist, ${ }^{42}$ which was revised by the research team (LS, DJ, MM, DK). The framework has five sections: (a) identifying the research question, (b) identifying relevant studies, (c) identifying the study selection criteria, (d) charting the data incorporating both quantitative and qualitative thematic analysis and (e) collating, summarising and reporting the results. 


\section{Review stages}

Stage 1: identifying the research question

Scoping reviews are a form of knowledge synthesis that present a broad overview of the evidence on a topic of interest, without addressing study quality, and can be used to identify key concepts for a topic area and identify any knowledge gaps. ${ }^{43}$ The concepts underpinning a research area can be mapped by systematically searching, selecting and synthesising existing knowledge. ${ }^{39} 44$

The primary research question is:

i. What is the extent of reported research on establishing VCoPs in healthcare (for clinical purposes) published in the last 10 years (2010 to current)?

Secondary research questions add focus to the review and provide guidance for setting up and conducting a VCoP for falls prevention:

ii. What methods are used to establish and maintain VCoPs (what frameworks are used for VCoP development, who are the participants, how is it coordinated and what are the methods of communication and knowledge exchange?).

iii. What potential barriers and facilitators are identified during the implementation of VCoPs?

The authors are aware and prepared for themes and recommendations that arise from the literature that are beyond these research questions and will amend and update the questions as required.

\section{Stage 2: identifying relevant studies}

\section{Eligibility criteria}

In a scoping review, the three elements of population, concept and context are used to establish inclusion and exclusion criteria. The population details the relevant characteristics of participants, the concept is the principal focus of the review and the context describes the setting under examination.

\section{Participants}

The population of interest is any healthcare professionals who are part of a VCoP for the purposes of building and exchanging knowledge, developing individual capabilities, ensuring their practice is evidence-based and enhancing interprofessional collaboration.

\section{Concept}

The concept is VCoPs for the purposes of improving clinical outcomes. CoPs that describe themselves as 'virtual', 'online' or 'web-based' are included. They should report on the establishment and maintenance of VCoPs that have been implemented in a healthcare setting for health professionals.

\section{Context}

The context is any platform used by healthcare professionals to support virtual interactions in healthcare for knowledge advancement and sharing of ideas. VCoPs that are purely for teaching purposes, for example, online learning, will be excluded.
Types of evidence sources

To be included, articles should be peer-reviewed and in the English language. Included articles can be existing literature on VCoPs including primary research studies of any design (quantitative, qualitative and mixed-methods), systematic reviews, meta-analyses, guideline implementation. Exclusions include grey literature, commentaries and any other opinion pieces. The articles need to be accessible as full text and published between January 2010 and October 2020.

\section{Search strategy}

A three-step approach will be developed by the study group in collaboration with an academic librarian. The librarian will execute the searches on behalf of the study group.

i. There will be an initial limited search of PubMed and Cumulative Index to Nursing and Allied Health Literature (CINAHL), to identify relevant studies to assist with search term development, based on the research questions and purpose of the study. The librarian will assist in guiding a rigorous analysis process to identify the best search terms and strategy related to VCoPs in healthcare. The process will be iterative, to ensure all relevant search terms are captured.

ii. Words in the title and abstract of the initial retrieved papers and indexing terms will be analysed and used to classify the articles.

iii. A second comprehensive search across PubMed, CINAHL, CENTRAL, PsycINFO, Cochrane Library and Education Resources Information Center from January 2010 to October 2020 will be conducted, to ensure VCoPs are contemporary in terms of design and content. The reference lists of all identified reports and articles will be searched for additional studies meeting the inclusion criteria. We will retrieve all supplementary files that are referred to in the included papers and any papers that are referred to in a particular study that were part of the research project.

Online supplemental appendix 1 shows the initial search strategy to be executed in CINAHL and PubMed.

\section{Stage 3: study selection}

All studies identified from the search strategy will be uploaded to the online systematic review software, Covidence. ${ }^{45}$ Two reviewers will independently screen the titles and abstracts of retrieved papers. The full texts of identified papers will be obtained and assessed by two independent reviewers, to identify studies that meet the inclusion criteria. Discrepancies will be resolved through discussion and if necessary, consensus will be achieved via a third reviewer. The results of the search will be presented in a PRISMA-ScR flow diagram (see figure 1). ${ }^{42}$

\section{Stage 4: data charting}

Data from eligible studies will be charted independently by two researchers using a data extraction 


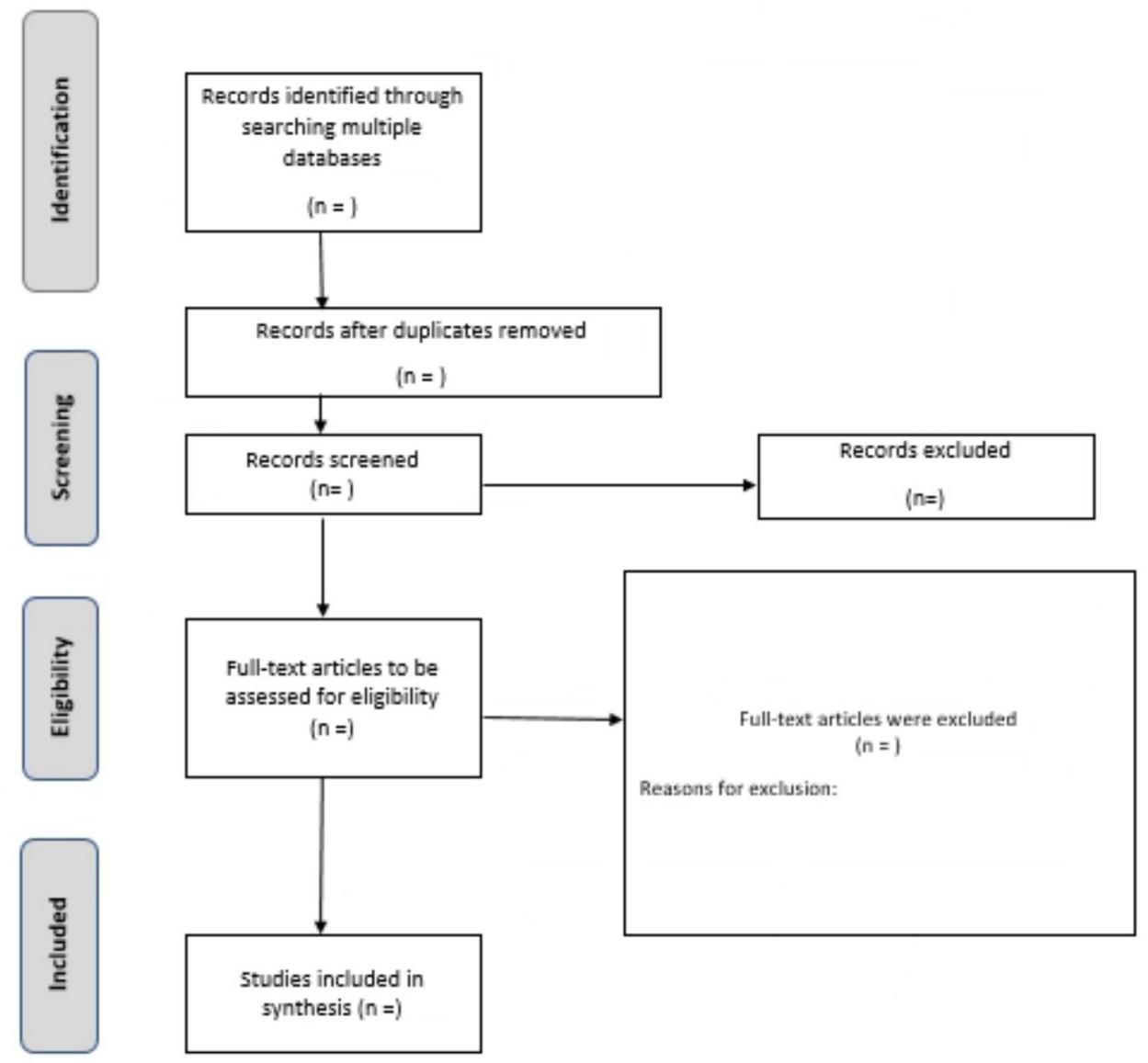

Figure 1 PRISMA-ScR flow diagram example. PRISMA-ScR, Preferred Reporting Items for Systematic reviews and MetaAnalyses extension for Scoping Reviews.

chart developed in Covidence. ${ }^{45}$ The chart will capture the relevant information on key study characteristics (eg, year of publication, country of origin, type of research, setting, study population of those in the VCoP), objectives, terminology used, development (activities undertaken at the inquiry, design and launch stages), barriers and facilitators to VCoP development, outcomes and key findings related to the review questions. This process will be iterative and variables may be identified following complete review of the full texts. The data extraction form will be trialled by two reviewers on a random sample of 10 included articles to ensure that all relevant results were able to be captured, and modifications will be made as required. After this, the same two reviewers will independently chart the data for all included studies, and then compare and merge the data into a final dataset. Conflicts at the data merging stage will be resolved by discussion until consensus is reached. If a consensus cannot be reached, a third study group member will be consulted.

\section{Stage 5: collating, summarising and reporting the results}

The synthesis of extracted data will include thematic analysis for qualitative data. Quantitative data will be summarised using frequency analysis, with the counts and percentages of articles for each category calculated. Data synthesis will be an iterative process with new categories and themes identified through ongoing analysis. For the qualitative analysis, two reviewers will categorise the key components independently in Excel. Through discussion, they will develop a coding framework. The coding framework will be piloted on a random sample of 10 included articles by the two primary reviewers and modifications will be made as required. One of the primary reviewers will then code the remaining articles according to the final framework. Quantitative results will be summarised in tables, charts and diagrams as indicated by the data, to allow for easy comparison. Following synthesis and analysis of the data best practice methods to establish and maintain VCoPs, barriers and facilitators to establishing VCoPs, approaches to evaluation and the impact of VCoPs on clinical practice, will be identified.

\section{Ethics and dissemination}

The results of this scoping review will highlight the best ways to design and manage VCoPs in healthcare organisations. The findings will be presented at relevant stakeholder workshops, conferences and published in peer-reviewed journals. Ethics approval is not required for this scoping review. 


\section{SUMMARY}

VCoPs are becoming increasingly popular, yet the best methods of how to establish them have not been realised. The proposed scoping review will follow an updated, five-step rigorous methodology for conducting scoping reviews as described by the Joanna Briggs Institute. The review will provide a new and detailed analysis of the extent of the literature on VCoPs in healthcare published in the last 10 years. It will highlight the best methods for establishing and maintaining VCoPs within a healthcare setting. It will also outline any potential barriers and facilitators to developing a VCoP in a healthcare setting. The findings will inform the development of a standardised but flexible framework for the translation of falls prevention clinical guidelines into practice.

\section{Twitter Debra Kiegaldie @DKiegaldie}

Contributors LS was involved in study conception, preliminary literature review, writing and editing of the protocol, scoping review framework and analysis, design of the search strategy and content expert input. DJ was involved in study conception, editing of the protocol, content expert input and preliminary literature review. MM and DK were involved in editing of the protocol, provided general guidance to the research team, were involved in study conception and content expert input. All authors have made substantive intellectual contributions to the development of this protocol. All authors read and approved the manuscript.

Funding This scoping review is being conducted as a part of an NHMRC funded public-private partnership (\#GNT1152853) which aims to use implementation science principles to enable both clinicians and patients to better mitigate future risk of hospital falls and to reduce falls rates. The partnership is between the Healthscope private hospital network, Holmesglen Institute and Australian universities

\section{Competing interests None declared.}

Patient consent for publication Not required.

Provenance and peer review Not commissioned; externally peer reviewed.

Supplemental material This content has been supplied by the author(s). It has not been vetted by BMJ Publishing Group Limited (BMJ) and may not have been peer-reviewed. Any opinions or recommendations discussed are solely those of the author(s) and are not endorsed by BMJ. BMJ disclaims all liability and responsibility arising from any reliance placed on the content. Where the content includes any translated material, BMJ does not warrant the accuracy and reliability of the translations (including but not limited to local regulations, clinical guidelines, terminology, drug names and drug dosages), and is not responsible for any error and/or omissions arising from translation and adaptation or otherwise.

Open access This is an open access article distributed in accordance with the Creative Commons Attribution Non Commercial (CC BY-NC 4.0) license, which permits others to distribute, remix, adapt, build upon this work non-commercially, and license their derivative works on different terms, provided the original work is properly cited, appropriate credit is given, any changes made indicated, and the use is non-commercial. See: http://creativecommons.org/licenses/by-nc/4.0/.

\section{ORCID iDs}

Louise Shaw http://orcid.org/0000-0001-8188-2313

Meg Morris http://orcid.org/0000-0002-0114-4175

\section{REFERENCES}

1 Wenger E, McDermott RA, Snyder W. Cultivating communities of practice: a guide to managing knowledge. Brighton, Massachusetts: Harvard Business Press, 2002.

2 Wenger E. Communities of practice: a brief introduction, 2004. Available: https://wenger-trayner.com/introduction-to-communitiesof-practice/

3 Nicolini D, Scarbrough H, Gracheva J. Communities of practice and situated learning in health care. The Oxford handbook of health care management 2016:255-78.
4 Kislov R. From a project team to a community of practice? an exploration of boundary and identity in the context of healthcare collaboration. Patient-Centred Health Care. Berlin: Springer, 2013: 103-17.

5 Barwick MA, Peters J, Boydell K. Getting to uptake: do communities of practice support the implementation of evidence-based practice? J Can Acad Child Adolesc Psychiatry 2009;18:16.

6 Piat M, Briand C, Bates E, et al. Recovery communities of practice: an innovative strategy for mental health system transformation. Psychiatr Serv 2016;67:10-12.

7 New South Wales Health. Communities of practice for the management of COVID19, 2020. Available: https://www.health.nsw. gov.au/Infectious/covid-19/communities-of-practice/Pages/default. aspx

8 LC L, Grimshaw JM, Nielsen C. Use of communities of practice in business and health care sectors: a systematic review. Implementation science 2009;4:1-9.

9 Ranmuthugala G, Cunningham FC, Plumb JJ, et al. A realist evaluation of the role of communities of practice in changing healthcare practice. Implement Sci 2011;6:49.

10 Fung-Kee-Fung M, Morash R, Goubanova E. Evaluating cops in cancer surgery. Handbook of research on communities of practice for organizational management and networking: methodologies for competitive advantage. Pennsylvania: IGI global, 2011: 456-66.

11 Barnett S, Jones SC, Bennett S, et al. A virtual community of practice for general practice training: a preimplementation survey. JMIR Med Educ 2016;2:e13.

12 Dieleman C, Duncan EAS. Investigating the purpose of an online discussion group for health professionals: a case example from forensic occupational therapy. BMC Health Serv Res 2013;13:1-8.

13 Mills J, Li C, Fullerton S, et al. Staying connected and informed: online resources and virtual communities of practice supporting palliative care during the novel coronavirus pandemic. Prog Palliat Care 2020;28:251-3.

14 Dubé L, Bourhis A, Jacob R. Towards a typology of virtual communities of practice. IJIKM 2006;1:069-93.

15 Barnett S, Jones SC, Bennett S, et al. General practice training and virtual communities of practice - a review of the literature. BMC Fam Pract 2012;13:1-12.

16 Antonacci G, Fronzetti Colladon A, Stefanini A, et al. It is rotating leaders who build the Swarm: social network determinants of growth for healthcare virtual communities of practice. Journal of Knowledge Management 2017;21:1218-39.

17 Rolls K, Kowal D, Elliott D, et al. Building a statewide knowledge network for clinicians in intensive care units: knowledge brokering and the NSW intensive care coordination and monitoring unit (ICCMU). Aust Crit Care 2008;21:29-37.

18 Ho K, Jarvis-Selinger S, Norman CD, et al. Electronic communities of practice: guidelines from a project. J Contin Educ Health Prof 2010;30:139-43.

19 McLoughlin C, Patel KD, O'Callaghan T, et al. The use of virtual communities of practice to improve interprofessional collaboration and education: findings from an integrated review. J Interprof Care 2018;32:136-42.

20 Pan SL, Leidner DE. Bridging communities of practice with information technology in pursuit of global knowledge sharing. The Journal of Strategic Information Systems 2003;12:71-88.

21 DeSanctis G, Fayard A-L, Roach M, et al. Learning in online forums. European Management Journal 2003;21:565-77.

22 Hara N, Foon Hew K, Hew KF. Knowledge-sharing in an online community of health-care professionals. Info Technology \& People 2007;20:235-61.

23 Malinen S. Understanding user participation in online communities: a systematic literature review of empirical studies. Comput Human Behav 2015;46:228-38.

24 Amichai-Hamburger Y, Gazit T, Bar-llan J, et al. Psychological factors behind the lack of participation in online discussions. Comput Human Behav 2016;55:268-77.

25 Nistor N, Baltes B, Dascălu M, et al. Participation in virtual academic communities of practice under the influence of technology acceptance and community factors. A learning analytics application. Comput Human Behav 2014;34:339-44.

26 Ardichvili A. Learning and knowledge sharing in virtual communities of practice: motivators, barriers, and enablers. Advances in Developing Human Resources 2008;10:541-54.

27 Ardichvili A, Page V, Wentling T. Motivation and barriers to participation in virtual knowledge-sharing communities of practice. Journal of Knowledge Management 2003;7:64-77.

28 Francis-Coad J, Etherton-Beer C, Bulsara C, et al. Can a web-based community of practice be established and operated to lead falls prevention activity in residential care? Geriatr Nurs 2017;38:133-40. 
29 Sarkies MN, Bowles K-A, Skinner EH, et al. The effectiveness of research implementation strategies for promoting evidence-informed policy and management decisions in healthcare: a systematic review. Implement Sci 2017;12:132.

30 Elshaug AG, Watt AM, Mundy L, et al. Over 150 potentially lowvalue health care practices: an Australian study. Medical Journal of Australia 2012;197:556-60.

31 Haines TP, Bowles K-A, Mitchell D, et al. Impact of disinvestment from weekend allied health services across acute medical and surgical wards: 2 stepped-wedge cluster randomised controlled trials. PLoS Med 2017;14:e1002412.

32 Kredo T, Bernhardsson S, Machingaidze S, et al. Guide to clinical practice guidelines: the current state of play. Int J Qual Health Care 2016;28:122-8.

33 Shekelle P, Woolf S, Grimshaw JM, et al. Developing clinical practice guidelines: reviewing, reporting, and publishing guidelines; updating guidelines; and the emerging issues of enhancing guideline implementability and accounting for comorbid conditions in Guideline development. Implement Sci 2012;7:62.

34 Runciman WB, Hunt TD, Hannaford NA, et al. CareTrack: assessing the appropriateness of health care delivery in Australia. Medical Journal of Australia 2012;197:100-5.

35 Greenhalgh T, Howick J, Maskrey N, et al. Evidence based medicine: a movement in crisis? BMJ 2014;348:g3725.

36 Pronovost PJ. Enhancing physicians' use of clinical guidelines. JAMA 2013;310:2501-2.
37 Ford J, Korjonen H, Keswani A, et al. Virtual communities of practice: can they support the prevention agenda in public health? Online $J$ Public Health Inform 2015;7:e222.

38 Francis-Coad J, Etherton-Beer C, Bulsara C, et al. Can a webbased community of practice be established and operated to lead falls prevention activity in residential care? Geriatr Nurs 2017;38:133-40.

39 Arksey H, O'Malley L. Scoping studies: towards a methodological framework. Int J Soc Res Methodol 2005;8:19-32.

40 Peters MDJ, Godfrey CM, Khalil H, et al. Guidance for conducting systematic scoping reviews. Int J Evid Based Healthc 2015;13:141-6.

41 Peters MDJ GC, Mclnerney P, Munn Z. Chapter 11: scoping reviews (2020 version). In: Aromataris EMZ, ed. JBI manual for evidence synthesis. Adelaide, Australia: Joanna Briggs Institute, 2020.

42 Tricco AC, Lillie E, Zarin W, et al. PRISMA extension for scoping reviews (PRISMA-ScR): checklist and explanation. Ann Intern Med 2018;169:467-73.

43 Peters MDJ, Marnie C, Tricco AC, et al. Updated methodological guidance for the conduct of scoping reviews. JBI Evid Synth 2020;18:2119-26.

44 Colquhoun HL, Levac D, O'Brien KK, et al. Scoping reviews: time for clarity in definition, methods, and reporting. $J$ Clin Epidemiol 2014:67:1291-4.

45 Veritas Health Innovation. Covidence systematic review software. Melbourne, Australia 2019. 